\title{
Influence des clairières forestières sur la répartition spatiale des grands mammifères dans la forêt dense du Bassin du Congo: cas du Parc National de Boumba-Bek (Sud-Est Cameroun)
}

\author{
Paul NOUPA ${ }^{1^{*}}$ et Bernard-Aloys NKONGMENECK ${ }^{2,3}$ \\ ${ }^{1}$ Union Internationale pour la Conservation de la Nature (UICN), B.P. 5506 Yaoundé, Cameroun. \\ ${ }^{2}$ Laboratoire de Systématique et d'Ecologie, Département de Biologie et de Physiologie Végétales, Université \\ de Yaoundé I, Cameroun. \\ ${ }^{3}$ Musée d'Ecologie du Millénium (MEM) de Yaoundé, B.P. 8038 Yaoundé, Cameroun. \\ *Auteur correspondant, Tel: (237) 9964 16 46,E-mail: paul.noupa@iucn.org
}

\section{RESUME}

Les clairières forestières sont des ouvertures "naturelles" dans la canopée de la forêt dense. Elles sont dominées par une végétation majoritairement composée d'herbacées. Elles constituent des écosystèmes particuliers dont les influences sur la concentration de la population animale sont encore mal connues. Les formations herbeuses représentent seulement $1 \%$ de la superficie du massif forestier dans le Bassin du Congo. La présente étude vise à déterminer le rôle que les clairières forestières exercent sur la population animale. L'étude a été menée en faisant des inventaires fauniques linéaires pluridirectionnels sur une distance de $5 \mathrm{~km}$ à partir du centre des clairières de Likolo et Batouka. Un gradient des traces animales a été ensuite calculé pour déterminer l'influence des clairières sur les populations animales. Le gradient des traces dans la clairière de Likolo est défini par l'équation $\mathrm{Y}=0,0661 \mathrm{e}^{-1,3399 \mathrm{x}}$ et $\mathrm{Y}=0,0141 \mathrm{e}^{-0,884 \mathrm{x}}$ pour la clairière de Batouka. Il se dégage de l'analyse que les animaux ne sont pas uniformément répartis dans le massif forestier autour des clairières forestières. La présence des clairières forestières de Likolo et Batouka provoque une concentration animale six fois plus grande en son centre que dans le reste du massif aux alentours dans un rayon de 5 Kilomètres à Likolo et quatre fois plus grande en son centre que dans le reste du massif forestier aux alentours à Batouka. Les clairières forestières de Likolo et Batouka sont des pôles de concentration animale dans le Parc National de Boumba-Bek.

(c) 2008 International Formulae Group. All rights reserved.

Mots clés: Ecosystème; Clairières forestières; Aménagement de la faune; Parc National de Boumba-Bek; SudEst Cameroun; Bassin du Congo.

\section{INTRODUCTION}

Dans le massif forestier du Bassin du Congo, on rencontre fréquemment des ouvertures naturelles dans la canopée et les espaces ainsi ouverts sont pour la plupart couverts d'une végétation majoritairement dominée d'herbacées (Noupa et Kouahou, 2000). Ces ouvertures qui sont de diverses natures, sont connues sous le nom de « clairières forestières » ou de «savanes intra forestières » (Photo 1). Elles constituent des écosystèmes particuliers dont les influences sur la concentration de la population animale sont encore mal connues même si les observations et les affirmations empiriques semblent confirmer un certain effet. Elles entretiennent la population des herbivores dans le massif et l'ensemble de la population des autres espèces animales au bénéfice des interrelations complexes de la chaîne trophique. Selon Letouzey (1985) cité par Stromayer et Ekobo (1991), les formations herbeuses en milieu forestier représentent seulement $1 \%$ de la superficie du massif forestier dans le Bassin du Congo et pourtant, elles détermineraient à elles seules le succès ou l'échec des actions de conservation de la riche diversité biologique animale qu'on 
reconnaît à ces massifs de forêt dense. La présente étude vise à déterminer l'impact de ces formations herbeuses sur la faune, en d'autres termes le rôle attractif que les savanes intra forestières exercent sur la population animale, rôle qui a été comparé à celui d'un aimant sur les populations animales par Solopek (2000). La bonne compréhension de cet impact est déterminante pour l'aménagement de la faune dans l'ensemble du massif forestier du Bassin du Congo.

\section{MATERIEL ET METHODES \\ Localisation de la zone d'étude}

La zone d'étude, le massif de BoumbaBek (d'une superficie de 238.255 Ha; Décret N $^{\circ}$ 2005/3284/PM du 6 octobre 2005 portant création du Parc National de Boumba-Bek) s'étend entre les longitudes $15^{\circ} 35^{\prime} \mathrm{E}$ à $15^{\circ} 50^{\prime} \mathrm{E}$ et les latitudes $2^{\circ} 09^{\prime} \mathrm{N}$ à $2^{\circ} 20^{\prime} \mathrm{N}$ (figure 1). Le climat est typiquement équatorial avec quatre saisons. Les précipitations s'étalent tout au long de l'année avec deux pics en avril et en octobre (Harrison et Agland, 1987; Ekobo, 1995). La hauteur maximale des précipitations est d'environ $1500 \mathrm{~mm} / \mathrm{an}$. La grande saison des pluies s'étale de septembre à novembre alors que la petite va de mars à juin. La grande saison sèche dure de décembre à février et la petite de juillet à août. Les moyennes mensuelles de températures varient de $23,1{ }^{\circ} \mathrm{C}$ à $25^{\circ} \mathrm{C}$. $\mathrm{La}$ température annuelle moyenne est de $24{ }^{\circ} \mathrm{C}$. L'humidité relative de l'air varie de 60 à $90 \%$. Des taux de saturation peuvent être enregistrés pendant les nuits de saison sèche. Le système hydrographique fluvial coule vers le Sud jusqu'à la rivière Ngoko, un affluent du fleuve Congo.

$\mathrm{Du}$ point de vue géologique la région de Boumba-Bek est située sur le versant nordouest de la cuvette congolaise avec des formations métamorphiques typiques d'âge Précambrien à Cambrien appartenant à la série du Dja inférieur. Ces formations métamorphiques sont essentiellement composées de tillites, quartzites, schistes, calcaires et dolérites. Le socle de BoumbaBek est d'origine précambrienne, constitué en une base cristalline de granites et de roches métamorphiques recouvertes de schistes et de pierres à chaux et quartzite de grès appelé série basse du Dja (Laclavère, 1979 ; Ekobo, 1998).

Les sols de la zone appartiennent au sous-ordre des sols ferrallitiques typiques définis par une séparation du fer et de l'oxyde d'alumine. Ils sont argileux et la fine couche d'humus superficiels contient peu de matière organique. Ils sont acides et pauvres en azote et bases échangeables.

Dans cette région, le Gouvernement du Cameroun développe une stratégie de gestion durable des ressources naturelles par la création et l'aménagement de trois parcs nationaux (Lobéké, Boumba-Bek et Nki soient environ $6000 \mathrm{~km}^{2}$ ), et le développement d'une zone d'utilisation multiple autour de ces noyaux de conservation (Harrison et Agland, 1987).

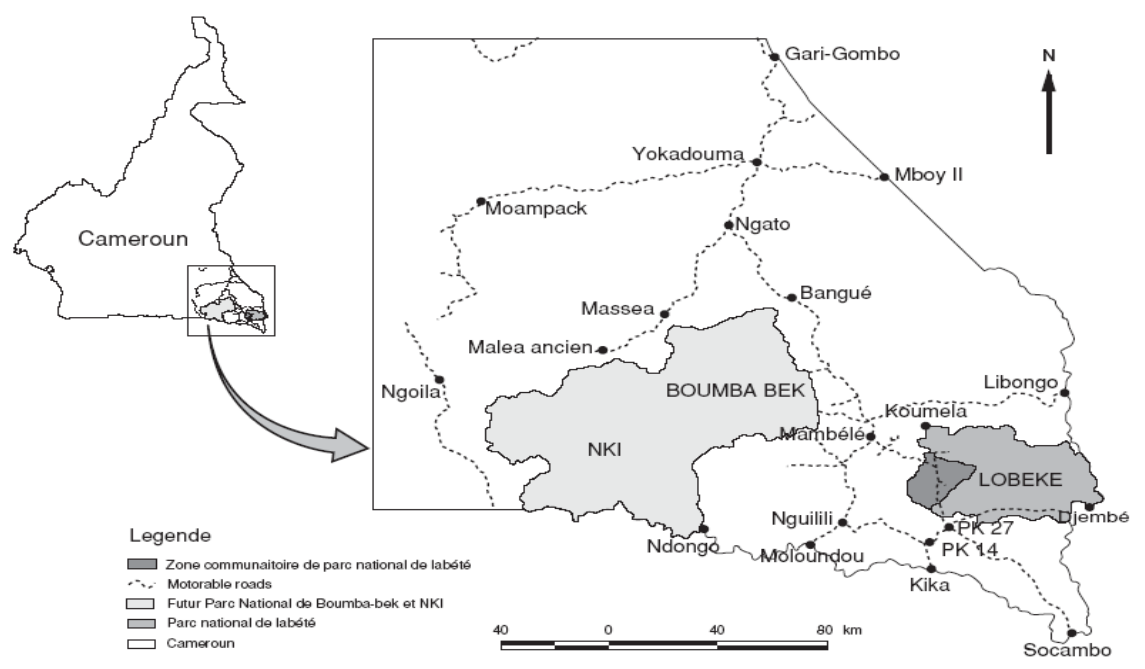

Figure 1: Localisation de la zone d'étude 


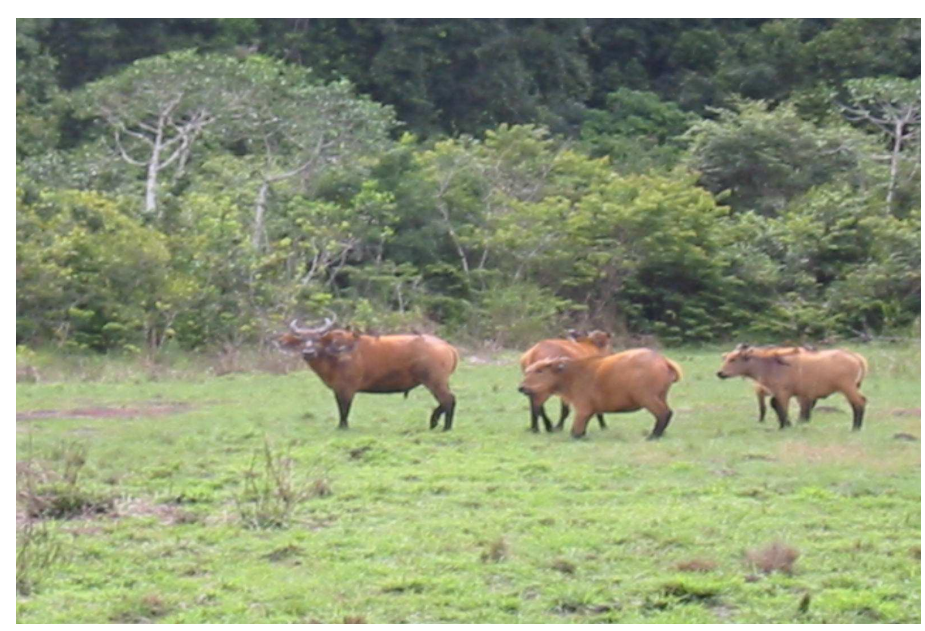

Photo 1 : Buffles dans la clairière forestière de Likolo (Parc National de Boumba-Bek, Sud-Est Cameroun).

\section{Présentation des clairières forestières}

Les clairières jouent un rôle dans le maintien de la diversité biologique. Elles sont de superficies variables allant de quelques mètres carrés à des centaines d'hectares. Elles s'installent sur des substrats pédologiques variés allant des sols profonds et marécageux aux sols maigres sur des affleurements rocheux. Letouzey (1985) les identifie comme étant des prairies développées sur Schistes chloriteux de la série précambrienne peu métamorphisée dite de Mbalmayo-Bengbis dont l'étude est encore très incomplète. Ces clairières sont caractérisées par une végétation à prédominance de la végétation herbacées dans laquelle la famille des graminées est à la fois la plus abondante et la plus dominante, ce qui leur confère leur rôle de pâturage naturel. L'espèce de graminée Loudetia simplex est la plus dominante et la plus abondante et de ce fait, elle influence la physionomie des clairières. Le déterminisme de formation et d'évolution des clairières est mal connu. Aubréville (1948) a assumé que les formations herbeuses des clairières forestières sont d'origine récentes et émanant de toute évidence de l'activité humaine. Mais Aubréville (1967) révisera ses convictions lorsqu'il comprit que ces formations particulières ne pouvaient pas résulter des interventions humaines seulement. Aussi postulat-il que les clairières forestières ou les savanes herbeuses des milieux forestiers représenteraient des reliques paléo climatiques. Letouzey (1985) affirme qu'aucune action humaine ancienne ne se révèle de façon évidente autour de ces formations et leur origine anthropique semble ne pas être retenue. Les clairières forestières restent influencées par la géologie locale et les conditions climatiques et pédologiques actuelles. Elles jouent des fonctions diverses aux communautés biologiques et constituent des écosystèmes dont le rôle écologique n'est pas entièrement exploré à ce jour. Les travaux d'inventaire botanique menés à Lobéké par l'équipe de Harris (1999) étaient étendus sur la reconnaissance globale de la végétation et n'ont pas permis une identification et une description effective des clairières forestières, celles-ci étant cependant reconnues d'importance par ces chercheurs. Cependant des explorations sont déjà faites dans le massif sur les fréquentations de ces clairières par la faune sauvage, notamment avec le dispositif de suivi écologique mis en place à Lobéke et les travaux de Ngoa (1999) portaient sur l'étude écologique de deux clairières (Djangui et Bolo), du parc national de Lobeké de la perspective de l'impact des activités anthropiques sur la faune sauvage. Du côté du massif forestier de Boumba-Bek et Nki, Nkongmeneck (1996) dans ses travaux a identifié 14 types d'écosystèmes parmi lesquels les clairières forestières qu'il affirme être des savanes intra forestières du fait de la 
présence des espèces caractéristiques de savane qu'on y rencontre. Ces travaux ont relevé l'intérêt de ces clairières forestières pour la diversité floristique du milieu suscitant alors un besoin de compléter les études de la richesse botanique du massif de Boumba-Bek par l'étude des groupes herbacées qu'on y rencontre. Ce besoin se trouvait renforcé par le rôle empiriquement évident d'attraction que les clairières forestières exerçaient sur les populations animales du massif forestier, rôle que tente de démontrer la présente publication.

Les clairières forestières seraient la résultante de l'influence de plusieurs facteurs complexes et dynamiques échelonnés dans le temps. Leurs origines sont variées et le déterminisme de leur formation est complexe. Elles sont dépendantes de divers facteurs et on peut citer par exemple :

- Les formations géologiques prédominantes,

- Les formations pédologiques sur lesquelles elles se sont développées,

- Les éléments du climat local.

- La composition phytosociologique dominante.

- L'activité humaine notamment avec l'élément feu de brousse qui a un impact sur la végétation de la clairière et qui est aussi décomposable en plusieurs facteurs (période de mise en feu, durée de la mise en feu, type et état de la végétation brûlée, ...).

\section{Méthodologie}

Pour étudier l'influence des clairières forestières sur la répartition spatiale des grands mammifères, deux clairières (Likolo et Batouka) les plus vastes et les plus fréquentées du massif connues à la date de l'étude, situées à 12 heures de marche des villages, ce qui leur assure une faible perturbation par les populations humaines ont été choisies. A partir du centre de chacune des clairières, les relevés des données ont été effectués sur des transects linéaires de $5000 \mathrm{~m}$ orientés suivant les directions Nord $\left(0^{\circ}\right)$, Nord-Est $\left(45^{\circ}\right)$, Est $\left(90^{\circ}\right)$, Sud-Est $\left(135^{\circ}\right)$, Sud $\left(180^{\circ}\right)$, Sud-Ouest $\left(225^{\circ}\right)$ Ouest $\left(270^{\circ}\right)$ et Nord-Ouest $\left(315^{\circ}\right)$. Les transects ainsi prédéfinis traversaient différentes strates de la végétation (clairière, forêt hétérogène, forêt marécageuse).
La méthodologie de base adoptée pour l'inventaire des mammifères est la "méthode de transects linéaires". Cette méthodologie a été largement utilisée dans le massif du sudest pour les différents inventaires de faune. Dans la réserve de faune du Dja, Usongo et Tchamba (1995), et Nzooh (2003) ont utilisé la méthode des transects linéaires. De même, Ekobo (1998), a utilisé la méthode dans l'inventaire des grands mammifères dans le massif de Boumba-Bek et Nki, et Bene et al. (2002) pour le suivi de la faune dans le même massif. D'autres auteurs ont fait large usage de la méthode ou en donnent la description et l'intérêt (Williams, 1996; Ngandjui, 1997 ; Ngnegueu, 1998; Sutherland, 1999) et une ample description de la méthodologie de transects linéaires se trouve dans de nombreuses documentations faisant autorité dans le domaine des inventaires en milieu forestier (White et Edwards, 2000). Suivant la méthode, toutes les traces sont notées sur des fiches standard. Les empreintes animales y compris les crottes, les nids et autres signes de présence sont identifiés le long du transect.

Dans l'analyse des données, le transect linéaire de 5000 mètres de long est divisé en 5 sous transects ou segment de 1000 mètres allant de 0 à $1000 \mathrm{~m}$, de 1000 à $2000 \mathrm{~m}$, de 2000 à $3000 \mathrm{~m}$, de 3000 à $4000 \mathrm{~m}$ et de 4000 à $5000 \mathrm{~m}$. Les densités de traces (en nombre de traces au $\mathrm{m}^{2}$ ) sur les différents segments des transects d'étude ont été calculées à partir des relevés obtenus dans les deux clairières. La densité des traces sur chaque sous transects a été calculée avec la formule de Rabinowitz (1997):

$$
D_{i j}=\frac{N_{i}}{2 X \bar{Y}_{i}},
$$

Où Dij est la densité (traces ou individus au $\mathrm{m}^{2}$ ) du transect $\mathrm{i}$ dans la strate $\mathrm{j}$. $\mathrm{X}$ est la longueur du transect ; $\mathrm{X}$ est fixé à $1000 \mathrm{~m} . \overline{Y i}$ est la moyenne des distances perpendiculaires en $\mathrm{m}$ dans le transect $\mathrm{i}$. Ni est le nombre de traces vues.

Une fois les densités des traces sur chaque transect calculées, la moyenne des densités dans la strate a été obtenue par la formule

$\overline{D_{j}}=\frac{\sum D_{i j}}{n}$,

Où $\mathrm{n}$ est le nombre de transect dans la strate j (8 transects).

Ensuite un gradient des traces animales est calculée par strate suivant la formule: 


$$
G_{j}=\frac{\overline{D_{j}}}{S_{j}},
$$

Où $S_{\mathrm{j}}$ est l'aire du cercle ou de l'anneau de rayon $1000 \mathrm{~m}$ définissant la strate $\mathrm{j}$.

La comparaison du gradient par strate permet de déterminer l'influence de la présence de la clairière forestière sur la répartition spatiale des mammifères dans le massif forestier. Ainsi, si le gradient est décroissant du centre de la clairière vers la périphérie, ceci indique que les traces d'animaux sont plus nombreuses dans le centre de la clairière qu'à la périphérie, indiquant par là-même que la concentration y est plus élevée qu'à la périphérie. La détermination de la valeur du gradient permettra ainsi de déterminer l'impact des clairières sur la concentration de la faune dans le massif de Boumba-Bek.

\section{RESULTATS}

Analyse globale des résultats de l'inventaire dans les clairières de Likolo et Batouka

Les densités de trace par azimut du transect et par segment calculées sont récapitulées dans le tableau 1 . Ces données de densité ont permis de calculer une densité moyenne par segment à partir de laquelle le gradient a été calculé suivant la formule de Rabinowitz (1997). Les valeurs des densités moyennes et les valeurs de gradient obtenues pour les deux clairières étudiées sont données dans le tableau 2.

Il s'en dégage que la densité des traces calculées dans les différents segments d'inventaire dans la clairière de Likolo suit un gradient qui décroît au fur et à mesure que l'on s'éloigne du centre de la clairière. Ceci indique que les animaux (toutes espèces confondues) ne sont pas uniformément répartis dans le massif forestier autour de la clairière forestière de Likolo. La présence de la clairière forestière de Likolo provoquerait donc une concentration animale six fois plus grande en son centre que dans le reste du massif forestier aux alentours dans un rayon de 5 Kilomètres. Ceci traduit l'influence que cette clairière exerce sur la répartition de la population animale dans le massif forestier adjacent.

De même, à Batouka, l'existence d'un gradient décroissant du centre vers la périphérie permet de conclure que les animaux (toutes espèces confondues) ne sont pas uniformément répartis dans le massif forestier autour de la clairière de Batouka. De même, la présence de cette clairière de Batouka provoque une concentration animale quatre fois plus grande en son centre que dans le reste du massif forestier aux alentours. Ces variations de la concentration des animaux du fait de la présence des clairières de Likolo et Batouka sont résumées par la figure 2.

Il a été procédé à un test de comparaison des moyennes des densités calculées sur les segments des différents transects étudiés à Likolo et à Batouka grâce au test de Student (test t) sur une distribution de type bilatéral. La comparaison des moyennes des densités s'est faite par pair sur l'hypothèse selon laquelle les moyennes de densités calculées dans les différents segments ne diffèrent pas significativement entre elles. Les résultats de l'analyse donnés dans les tableaux 3 et 4 sont les valeurs de probabilité associées à un test $\mathrm{t}$ de Student, avec une distribution bilatérale pour l'hypothèse que les moyennes des densités calculées dans les différents segments ne diffèrent pas significativement entre elles. Les valeurs de probabilité associées au test $\mathrm{t}$ sont partout inférieures à 0,5 exception faite entre les segments $\mathrm{C}$ et $\mathrm{D}$ dans les deux clairières Likolo et Batouka où ces valeurs sont respectivement de 0,78 à Likolo et 0,60 à Batouka. Dans les courbes de gradient ces segments marquent le fléchissement dans les tendances à la diminution du gradient, exprimant de ce fait le début de dilution de l'effet des clairières sur la concentration des animaux. Mais le test $\mathrm{t}$ pour la comparaison des moyennes confirme qu'il y a une différence significative du point de vue statistique sur les moyennes des densités des animaux calculées sur différents segments et par conséquent un gradient décroissant de distribution des traces animales du centre vers la périphérie des clairières.

Ceci se traduit par le fait que les traces des animaux sont plus importantes dans les segments proches du centre des clairières (dans le segment de 0 à 1000 mètres) qu'à la périphérie (Figure 3). Le gradient décroissant obtenu est caractérisé par sa fonction de variation $\mathrm{Y}$ et son coefficient de détermination R. 
P. NOUPA et B.-A. NKONGMENECK / Int. J. Biol. Chem. Sci. 2(2): 185-195, 2008

Tableau 1: Densités des traces d'animaux sur les segments des transects inventoriés dans les clairières de Likolo et Batouka (Nombre de Traces au m²).

\begin{tabular}{|c|c|c|c|c|c|c|c|c|c|c|c|c|c|c|c|c|}
\hline & \multicolumn{16}{|c|}{ Azimuth du Transect } \\
\hline & \multicolumn{2}{|c|}{$\mathbf{0}^{\circ}$} & \multicolumn{2}{|c|}{$45^{\circ}$} & \multicolumn{2}{|c|}{$90^{\circ}$} & \multicolumn{2}{|c|}{$135^{\circ}$} & \multicolumn{2}{|c|}{$180^{\circ}$} & \multicolumn{2}{|c|}{$225^{\circ}$} & \multicolumn{2}{|c|}{$270^{\circ}$} & \multicolumn{2}{|c|}{$315^{\circ}$} \\
\hline $\begin{array}{l}\text { Segment du calcul } \\
\text { de densité }\end{array}$ & Likolo & Batouka & Likolo & Batouka & Likolo & Batouka & Likolo & Batouka & Likolo & Batouka & Likolo & Batouka & Likolo & Batouka & Likolo & Batouka \\
\hline $\begin{array}{l}\text { Segment } 0 \text { à } 1000 \mathrm{~m} \\
\text { Segment } 1000 \text { à }\end{array}$ & 52329,89 & 3245 & 45534,21 & 16728,92 & 8751,48 & 8751,48 & 14943,5 & 72418,51 & 5655 & 32602,5 & 27232,17 & 3096,61 & 1576,27 & 1550 & 1065 & 6461,64 \\
\hline $\begin{array}{l}2000 \mathrm{~m} \\
\text { Segment } 2000 \text { à }\end{array}$ & 27259,86 & 1036 & 342 & 316,67 & 5285,21 & 5285,21 & 425 & 704 & 10594,74 & 808,73 & 20126,33 & 1547,61 & 382,05 & 382,05 & 2762,22 & 3876,67 \\
\hline $\begin{array}{l}3000 \mathrm{~m} \\
\text { Segment } 3000 \text { à }\end{array}$ & 15240 & 1769,23 & 1787,76 & 537,44 & 8112,09 & 8112,09 & 220,24 & 133,2 & 1272,57 & 836,15 & 2180,33 & 2702,5 & 660 & 660 & 2972,5 & 3672,79 \\
\hline $\begin{array}{l}4000 \mathrm{~m} \\
\text { Segment } 4000 \text { à }\end{array}$ & & 7210,84 & 235,29 & 1720,83 & 4173,08 & 4173,08 & 3583,75 & 1083,6 & 2894,37 & 1470,83 & 1234,78 & 435,88 & 1020 & 963,33 & 2338,33 & 5582,88 \\
\hline $5000 \mathrm{~m}$ & & 5419,12 & 163,64 & 2006,47 & 1265 & 16002,66 & 3048,68 & 0 & 623,08 & 5469,15 & 915,74 & 3422,4 & 195,12 & 3902,44 & 2735,09 & 5005,54 \\
\hline
\end{tabular}

Tableau 2: Densité moyenne par segment du transect et gradient calculé dans les clairières de Likolo et Batouka.

\begin{tabular}{lcccc} 
& \multicolumn{2}{c}{ Likolo } & Batouka \\
\cline { 2 - 5 } Segment du transect & $\begin{array}{c}\text { Densité Moyenne } \\
\text { Sur le Segment }\end{array}$ & $\begin{array}{c}\text { Gradient de densité } \\
\text { calculé à Likolo }\end{array}$ & $\begin{array}{c}\text { Densité Moyenne } \\
\text { Sur le Segment }\end{array}$ & $\begin{array}{c}\text { Gradient de densité } \\
\text { calculé à Batouka }\end{array}$ \\
\hline Segment 0 à 1000m & 19635,94 & 0,02501 & 18106,83 & 0,02307 \\
Segment 1000 à 2000m & 8397,18 & 0,00357 & 1744,62 & 0,00074 \\
Segment 2000 à 3000m & 4055,68 & 0,00086 & 2302,93 & 0,00049 \\
Segment 3000 à 4000m & 2211,37 & 0,00028 & 2830,16 & 0,00036 \\
Segment 4000 à 5000m & 1278,05 & 0,00011 & 5153,47 & 0,00044 \\
\hline
\end{tabular}


Tableau 3: Test de comparaison des moyennes des densités à Likolo.

\begin{tabular}{|c|c|c|c|c|c|}
\hline \multirow[b]{2}{*}{ Azimut } & \multicolumn{5}{|c|}{ Likolo } \\
\hline & $\begin{array}{l}\text { Segment } 0 \text { à } \\
1000 \mathrm{~m} \\
(\mathrm{~A})\end{array}$ & $\begin{array}{l}\text { Segment } 1000 \\
\text { à } 2000 \mathrm{~m} \\
(\mathrm{~B}) \\
\end{array}$ & $\begin{array}{l}\text { Segment } 2000 \\
\text { à } 3000 \mathrm{~m} \\
(\mathrm{C}) \\
\end{array}$ & $\begin{array}{l}\text { Segment } 3000 \\
\text { à } 4000 \mathrm{~m} \\
\text { (D) }\end{array}$ & $\begin{array}{l}\text { Segment } 4000 \\
\text { à } 5000 \mathrm{~m} \\
(\mathrm{E}) \\
\end{array}$ \\
\hline $0^{\circ}$ & 52329,89 & 27259,86 & 15240 & & \\
\hline $45^{\circ}$ & 45534,21 & 342 & 1787,76 & 235,29 & 163,64 \\
\hline $90^{\circ}$ & 8751,48 & 5285,21 & 8112,09 & 4173,08 & 1265 \\
\hline $135^{\circ}$ & 14943,5 & 425 & 220,24 & 3583,75 & 3048,68 \\
\hline $180^{\circ}$ & 5655 & 10594,74 & 1272,57 & 2894,37 & 623,08 \\
\hline $225^{\circ}$ & 27232,17 & 20126,33 & 2180,33 & 1234,78 & 915,74 \\
\hline $270^{\circ}$ & 1576,27 & 382,05 & 660 & 1020 & 195,12 \\
\hline $315^{\circ}$ & 1065 & 2762,22 & 2972,5 & 2338,33 & 2735,09 \\
\hline Moyenne & 19635,94 & 8397,17625 & 4055,68625 & 2211,371429 & 1278,05 \\
\hline Test $t(A, B)$ & 0,099417471 & & & & \\
\hline Test t $(\mathrm{B}, \mathrm{C})$ & & 0,153882289 & & & \\
\hline Test t $(C, D)$ & & & 0,789829158 & & \\
\hline Test t $(\mathrm{D}, \mathrm{E})$ & & & & 0,086852362 & \\
\hline Test t $(\mathrm{A}, \mathrm{C})$ & 0,042408181 & & & & \\
\hline Test t $(A, D)$ & 0,095480557 & & & & \\
\hline Test t $(\mathrm{A}, \mathrm{E})$ & 0,072815288 & & & & \\
\hline Test t $(\mathrm{B}, \mathrm{D})$ & & 0,267460139 & & & \\
\hline Test t $(\mathrm{B}, \mathrm{E})$ & & 0,178642231 & & & \\
\hline Test t $(\mathrm{C}, \mathrm{E})$ & & & 0,321372909 & & \\
\hline
\end{tabular}

Ainsi, la courbe de tendance de la variation du gradient des traces dans la clairière de Likolo est définie par l'équation $\mathrm{Y}=0,0661 \mathrm{e}^{-1,3399 \mathrm{x}}$ où $\mathrm{x}$ est la variable de distance. Le coefficient de détermination obtenu pour cette courbe de tendance est $\mathrm{R}^{2}=0,9783$, ce qui traduit le degré de rapprochement de la courbe de tendance de la perfection $\left(\mathrm{R}^{2}=1\right)$.

De même, la courbe de tendance de la variation du gradient des traces dans la clairière de Batouka est définie par l'équation $\mathrm{Y}=0,0141 \mathrm{e}^{-0,884 \mathrm{x}}$ où $\mathrm{x}$ est la variable de distance. Le coefficient de détermination pour cette courbe de tendance est $\mathrm{R}^{2}=0,6142$. Le coefficient de détermination étant supérieur à $50 \%$ pour les courbes de tendance de la diminution du gradient des densités dans les deux clairières, l'équation définie de la courbe de tendance traduit la réalité de la variation du gradient en fonction de la distance.

\section{Attraction des clairières sur les espèces animales}

Cette analyse a permis de démontrer le rôle des clairières dans le regroupement de la population de la faune. Les clairières sont des points de concentration de la population de la faune. Du dépouillement des fiches de collecte des données, il est ressorti que les traces de 16 espèces ont été rencontrées dans les inventaires faits dans et autour des clairières de Likolo et Batouka. Le calcul des densités spécifiques pour ces différentes espèces en fonction du type de milieu (Clairière, forêt marécageuse ou Forêt hétérogène) a été réalisé et le résultat résumé dans la figure 4. Il en ressort des similitudes dans l'exploitation des milieux par les espèces animales. On peut ainsi distinguer le pic du genre Cephalophus spp. qui représente la densité cumulée de 4 espèces de Céphalophes: $C$. callipigus (Céphalophe de Peter), C. dorsalis (céphalophe Bay), C. nigrifrons (céphalophe à front noir), C. leucogaster (Céphalophe à ventre blanc). Ce sont des espèces craintives, qui constituent des proies faciles pour les prédateurs qui fréquentent les zones de forte concentration d'animaux. Pour cette raison, les Céphalophes évoluent préférentiellement dans les forêts hétérogènes aux alentours des clairières, ne faisant que des incursions 
Tableau 4: Test de comparaison des moyennes des densités à Batouka.

\begin{tabular}{|c|c|c|c|c|c|}
\hline \multirow[b]{2}{*}{ Azimut } & \multicolumn{5}{|c|}{ Batouka } \\
\hline & $\begin{array}{c}\text { Segment } 0 \text { à } \\
1000 \mathrm{~m} \\
\text { (A) }\end{array}$ & $\begin{array}{l}\text { Segment } 1000 \\
\text { à } 2000 \mathrm{~m} \\
(\mathrm{~B})\end{array}$ & $\begin{array}{l}\text { Segment } 2000 \\
\text { à } 3000 \mathrm{~m} \\
(\mathrm{C})\end{array}$ & $\begin{array}{l}\text { Segment } 3000 \\
\text { à } 4000 \mathrm{~m} \\
\text { (D) }\end{array}$ & $\begin{array}{c}\text { Segment } 4000 \\
\text { à } 5000 \mathrm{~m} \\
(\mathrm{E})\end{array}$ \\
\hline $0^{\circ}$ & 3245 & 1036 & 1769,230769 & 7210,843373 & 5419,117647 \\
\hline $45^{\circ}$ & 16728,91892 & 316,6666667 & 537,4358974 & 1720,833333 & 2006,465517 \\
\hline $90^{\circ}$ & 8751,477273 & 5285,208333 & 8112,089552 & 4173,076923 & 16002,66129 \\
\hline $135^{\circ}$ & 72418,51351 & 704 & 133,203125 & 1083,6 & 0 \\
\hline $180^{\circ}$ & 32602,5 & 808,7323944 & 836,1486486 & 1470,833333 & 5469,146341 \\
\hline $225^{\circ}$ & 3096,610169 & 1547,605634 & 2702,5 & 435,8823529 & 3422,4 \\
\hline $270^{\circ}$ & 1550 & 382,0454545 & 660 & 963,3333333 & 3902,439024 \\
\hline $315^{\circ}$ & 6461,644737 & 3876,666667 & 3672,794118 & 5582,875 & 5005,538462 \\
\hline Moyenne & 18106,83 & 1744,615644 & 2302,925264 & 2830,159706 & 5153,471035 \\
\hline Test $\mathrm{t}(\mathrm{A}, \mathrm{B})$ & 0,104387803 & & & & \\
\hline Test t (B,C) & & 0,179852779 & & & \\
\hline Test t (C,D) & & & 0,609543664 & & \\
\hline Test $t(D, E)$ & & & & 0,178362321 & \\
\hline Test t $(\mathrm{A}, \mathrm{C})$ & 0,121604114 & & & & \\
\hline Test $t(A, D)$ & 0,121604114 & & & & \\
\hline Test t (A,E) & 0,209482314 & & & & \\
\hline Test $t(B, D)$ & & 0,223378067 & & & \\
\hline Test t (B,E) & & 0,026758547 & & & \\
\hline Test t (C,E) & & & 0,017065536 & & \\
\hline
\end{tabular}

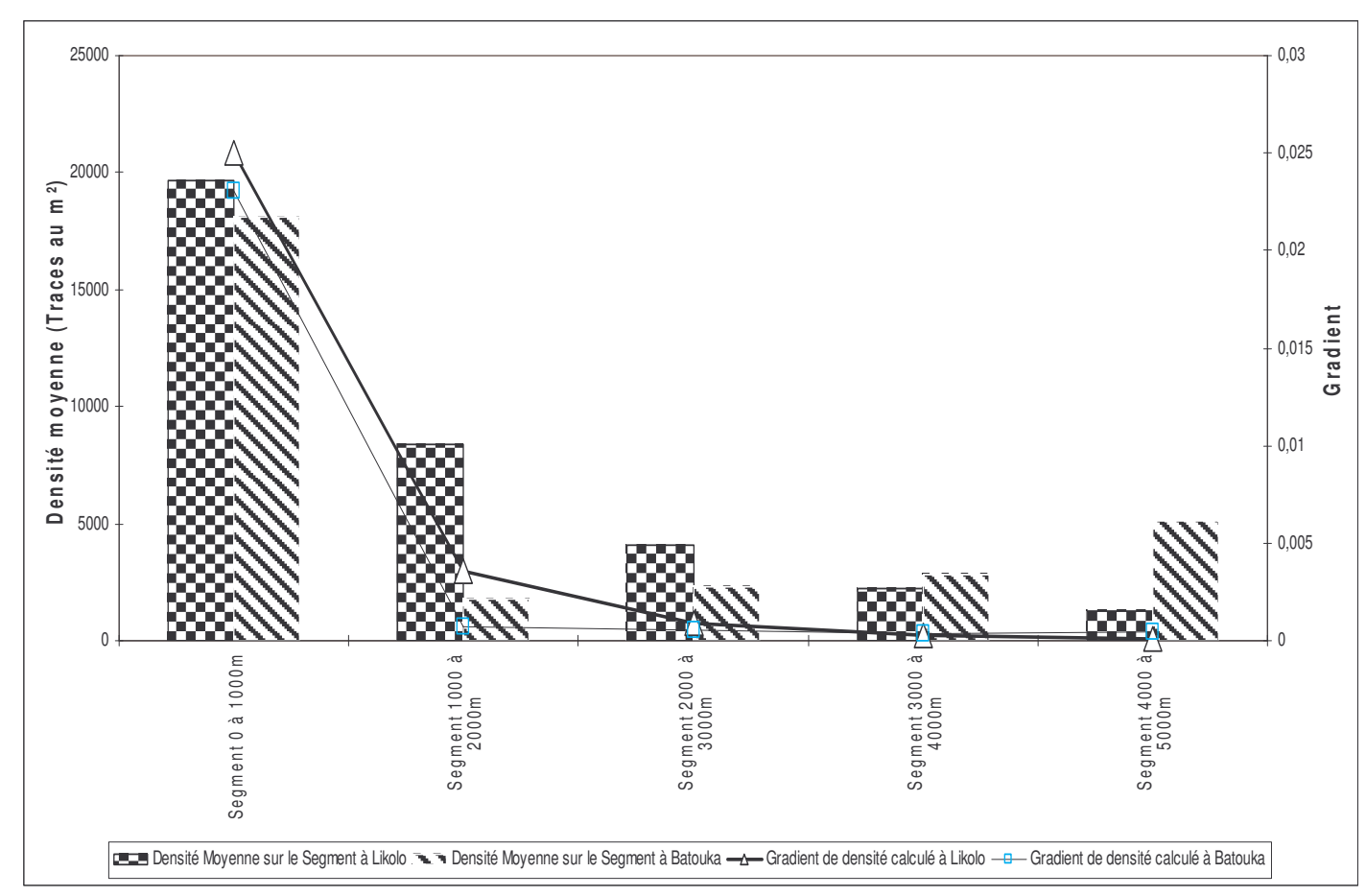

Figure 2: Variation de la concentration des traces d'animaux dans et autour des clairières d'étude. 


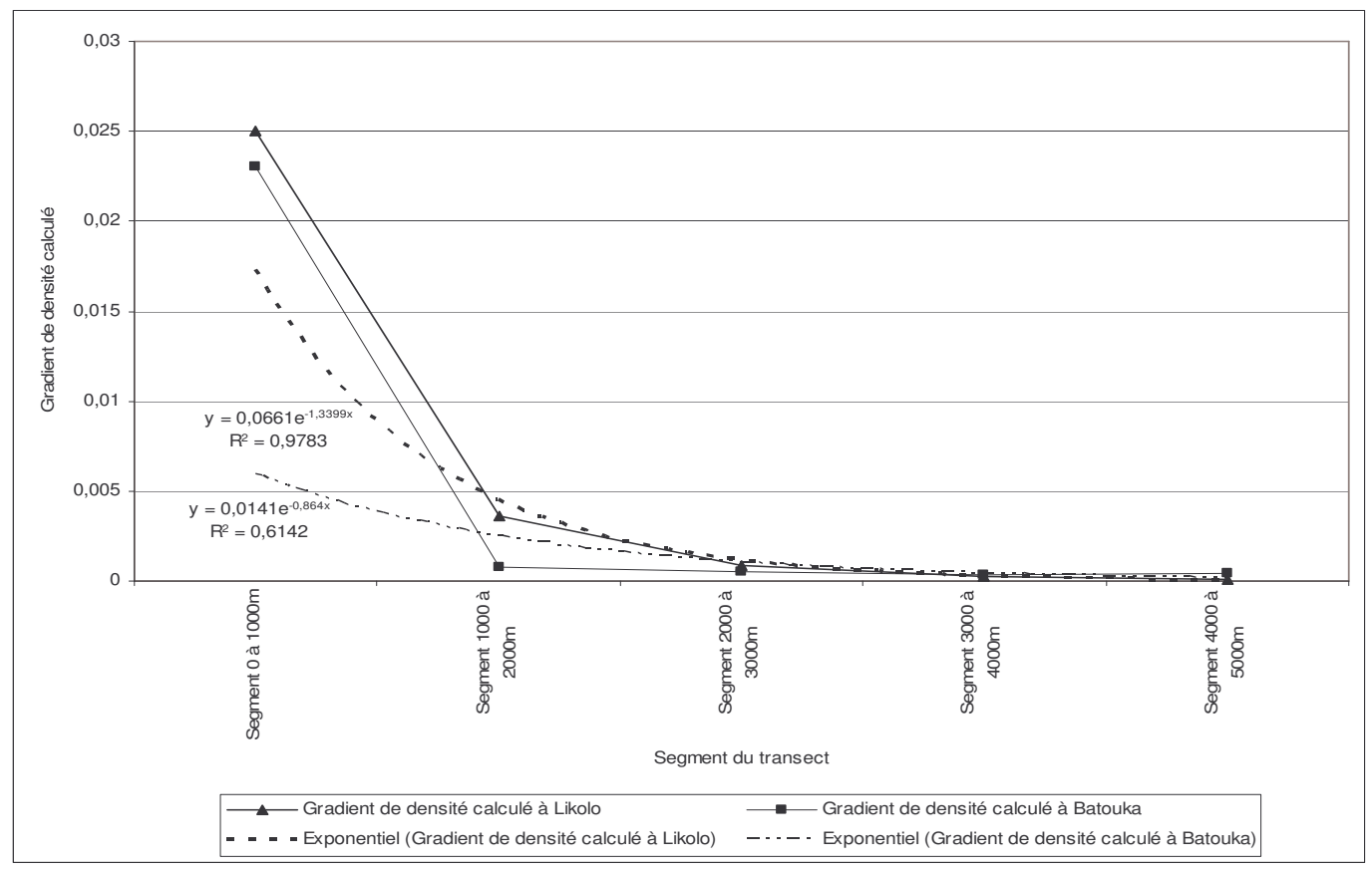

Figure 3: Gradient des traces dans les clairières de Likolo et de Batouka.

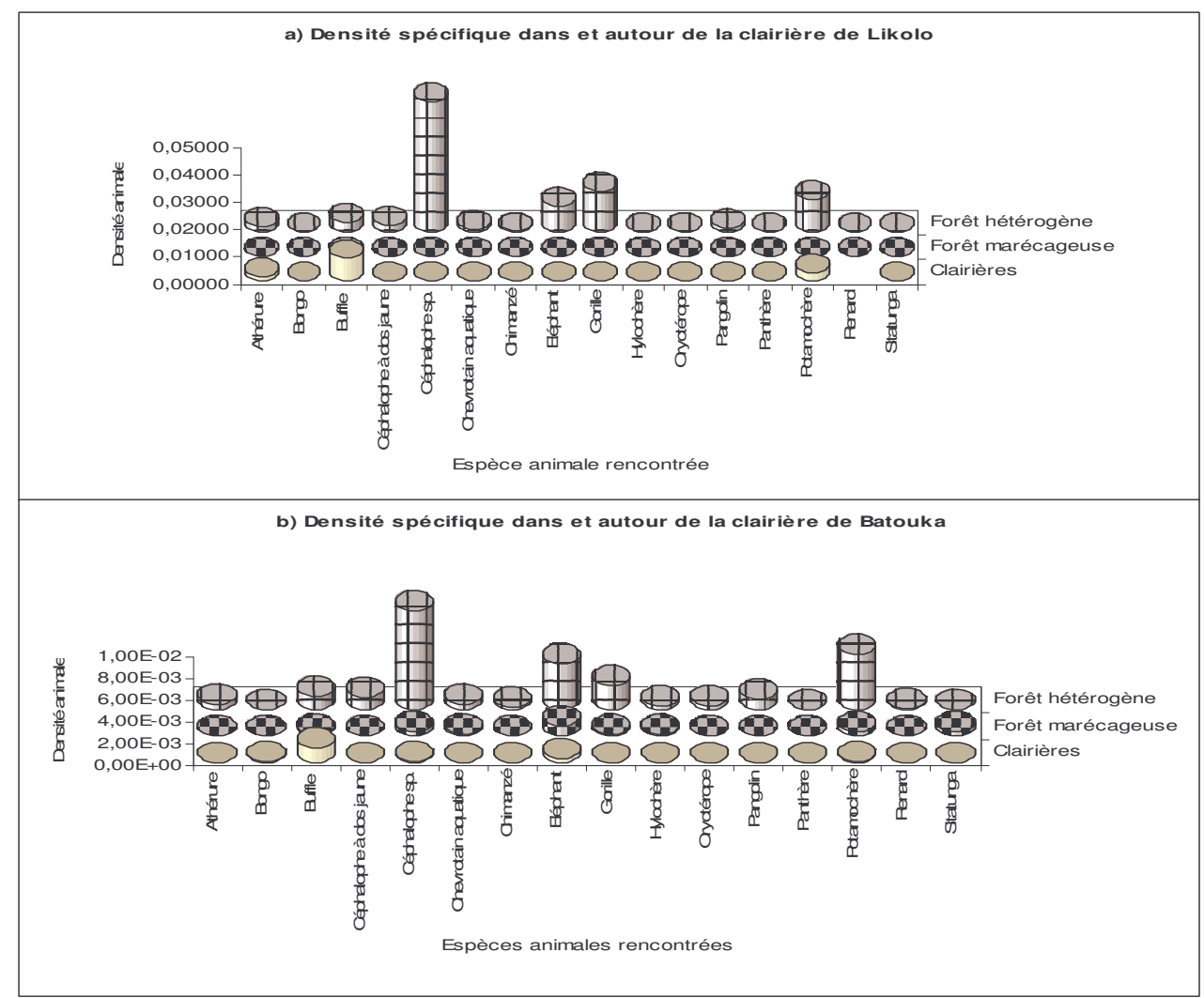

Figure 4: Densités spécifiques calculées dans les clairières de Likolo et Batouka. 
sporadiques pour brouter dans les clairières forestières. Les espèces Loxodonta africana (éléphant), Gorilla gorilla gorilla (gorille), et Potamochoerus porcus (potamochère), présentent aussi des densités élevées dans les forêts hétérogènes. Il s'agit en réalité des espèces ubiquistes qui affectionnent tous les habitats dans le milieu en fonction de leurs besoins. Ce qui n'est pas le cas du Syncerus caffer nanus (Buffle) et le (Tragelaphus euryceros) Bongo qui sont des espèces fortement inféodées aux clairières forestières où elles passent le maximum de leur temps, ne se déplaçant que pour la recherche des points d'eau lorsqu'il n'y en a pas dans les clairières. Les densités des ces espèces sont nettement élevées dans les clairières comparées à celles des autres espèces.

\section{DISCUSSION}

Il ressort de cette étude que les clairières forestières de Likolo et Batouka sont des pôles de concentration animale. Ceci confirme les résultats des travaux antérieurs menés dans ce massif. En effet, dans l'inventaire biologique du massif de BoumbaBek et Nki menée en vue de son classement en Parc National, Ekobo (1998) trouve que les densités des empreintes du Buffle (Syncerus caffer nanus), du sitatunga (Tragelaphus spekei), du bongo (Tragelaphus euryceros) et du leopard (Panthera pardus) sont plus élevées dans la partie de la forêt ayant les clairières que dans le reste du milieu environnant. De même, le rapport MIKE ("Monitoring of Illegal Killing of Elephant") du site de Boumba-Bek confirme que les clairières qui ne représentent que moins de $1 \%$ des différents types de végétation du milieu concentrent les plus grands indices de présence de nombreuses espèces dont l'éléphant avec entre 8974 et 10967 signes au $\mathrm{km}^{2}$ dans les clairières contre entre 1 et 2993 signes dans le reste du milieu environnant (Bene, 2004). Ces clairières jouent différents rôles dans l'éthologie des différentes espèces qui les fréquentent: arène nuptiale, lieu de broutage, lieu de repos, etc. Au bénéfice des interdépendances dans les relations alimentaires entre différentes espèces animales du milieu, les clairières forestières étant les uniques sites de broutage pour les herbivores, concentrent autour d'elles toute la complexité de la chaîne trophique du milieu forestier. Leurs rôles de pôle de concentration animale sont ainsi mis en évidence par la présente étude. Par ce fait cette étude pose les bases de la prise en compte de ces écosystèmes dans le développement de l'aménagement des massifs qui les contiennent. Leurs superficies, leurs distributions hasardeuses dans le massif et leurs capacités productives, toutes choses liées au hasard des conjonctures géologiques, pédologiques et climatiques ne sont-elles pas autant de facteurs naturels qui justifient le déterminisme des migrations de certaines espèces ? Le rôle des clairières forestières dans le suivi des populations des herbivores et de toute la hiérarchie dans la chaîne trophique du milieu contraste avec la fragilité et la répartition hasardeuse de ces écosystèmes dans le massif du bassin du Congo. Il est fort urgent qu'une étude sur la dynamique de ces écosystèmes particuliers à la base de la stabilité des populations animales dans le massif forestier du Bassin du Congo soit faite en vue de leur meilleure valorisation dans la perspective de l'aménagement de la faune. Ceci est d'autant plus opportun que le cadre de collaboration est en développement dans la sous région à l'issue du sommet des Chefs d'Etats de mars 1999 à Yaoundé pour optimiser les chances de sauvegarder une surface représentative d'habitat viable pour la faune sauvage dans un milieu en dégradation alarmante du fait de l'exploitation forestière et du développement des activités agricoles des populations rurales. Autant les zones humides font l'objet d'interventions spécifiques et particulières, autant, il est urgent que soit développé un projet «clairières forestières dans le bassin du Congo » pour développer une stratégie cohérente d'aménagement de ces écosystèmes qui sont les points de concentration animale et donc les centres vitaux au même titre que les points d'eau dans le massif, entendu qu'aucune évidence ne dément leur rôle dans le déterminisme des grands migrateurs. Les clairières forestières joueraient-elles les rôles de relais dans les échanges biologiques? Auquel cas il s'agirait aussi d'envisager dans les plans directeurs d'aménagement des massifs des dispositions spéciales pour les clairières situées en dehors des aires protégées, étant entendu que les $80 \%$ de la population animale de la région qui se retrouve en dehors des aires protégées trouvent en ces écosystèmes des sites vitaux indispensables pour leur survie. 


\section{BIBLIOGRAPHIE}

Aubréville A. 1948. Richesses et Misères des Forêts de l'Afrique Noire Française. Paris.

Aubréville A. 1967. Les étranges mosaïques forêt-savane du sommet de la boucle de l'Ogooué au Gabon. Adansonia 7: 13-22.

Bene BCL, Nzooh ZL, Usongo L. 2002. Résultats préliminaires du suivi écologique dans le Parc National de Boumba Bek. Rapport WWF CARPO. 25pp.

Bene BCL. 2004. Rapport inventaires en Afrique Centrale (2003-2004). Monitoring of the illegal killing of elephants (MIKE). WWF \& WCS. 49 p.

Ekobo A. 1995. Conservation of the African forest elephant (Loxodonta africana cyclotis) in Lobeke, Southeast Cameroon. $\mathrm{PhD}$ thesis, University of Kent, $151 \mathrm{pp}$.

Ekobo A. 1998. Large mammals and vegetation surveys in the Boumba-BekNki area. Report WWF CPO, 63pp + annexes.

Fouda EB. 1998. Dénombrement, densité et distribution de l'éléphant de forêt d'Afrique (Loxodonta africana) dans la zone du projet Boumba-Bek et Nki sudest Cameroun. Mémoire, FASA, Université de Dschang, 89p + annexes.

Gartlan S. 1989. La Conservation des Ecosystèmes Forestiers du Cameroun. UICN: Gland, Suisse et Cambridge, Royaume - Uni ; 1869.

Harris D. 1999. Lobéké: botanical inventory. Report for WWF Cameroon, 130p.

Harrisson M, Agland P. 1987. A draft proposal for the designation of three new national forest parks. Dja river Films, LTD.

Laclavère G. 1979. Atlas de la République Unie du Cameroun. Ed. Jeune Afrique: Paris; 72 pp.

Letouzey R. 1985. Notice de la carte phytogéographique $d u$ Cameroun au 1:500000. Institut de la Carte Internationale de la Végétation, Toulouse, France et IRA, Yaoundé, Cameroun ; 95142.

Ngandjui G. 1997. Inventaire et utilisation durable de la faune mammalienne en milieu forestier équatorial : cas du secteur Ouest de la réserve de la biosphère du Dja
(Sud-Cameroun). Thèse, Université Paul Valery - Montpellier III : 256p.

Ngnegueu PR. 1998. Exploitation de la faune sauvage dans la région du Dja (Sud-Est Cameroun). Communication to the Workshop on the sustainable hunting in the southeastern Cameroon. Ministère de la Recherche Scientifique du Cameroun, CIFOR et Coopération Française.

Ngoa EO. 1999. Etude écologique de deux clairières (Djangui et Bolo), du Parc National de Lobeké : Impact des activités anthropiques sur la faune sauvage. Mémoire, FASA, Université de Dschang, $59 p+$ annexes

Nkongmeneck BA. 1996. The Boumba-Bek and Nki Forest Reserve, Botany and Ethnobotany. WWF Cameroun ; 146 p.

Noupa P, E Kouahou. 2000. Identification et description des clairières forestières à l'intérieur du futur Parc National de Boumba-Bek et Nki (Sud-Est Cameroun). Rapport WWF Cameroun, Jengi South East Forest Programme, 60 p.

Nzooh DZL. 2003. Suivi écologique dans le Parc National de Lobeke et sa zone périphérique: données de base sur la dynamique des populations de grands et moyens mammifères et des activités anthropiques. Rapport WWF CARPO, 201pp.

Rabinowitz RA. 1997. Wildlife Field Research and Conservation Training Manual. WCS; 281p.

Usongo L, Tchamba M. 1995. Inventaire de faune dans la réserve de faune du Dja. Rapport WWF Cameroun.

Solopek P. 2000. Silence descends on Africa's forests: Wildlife and forests in peril. Chicago Tribune, March 2000.

Sutherland WJ. 1999. Ecological Census Techniques: a Handbook. Cambridge University Press.

Stromayer K, Ekobo A. 1991. Biological survey of the south-eastern Cameroon. Report WWF/CAR.

White L, Edwards A. 2000. Conservation en Forêt Pluviale Africaine: Méthodes de Recherche. Wildlife Conservation Society: New York; 444 pp.

Williams JS. 1996. Ecological Census Techniques: a Handbook. Cambridge Editions; 285 pp. 